\title{
The Determination of Sex.
}

By Prof. R. Goldschmidt, Kaiser Wilhelm-Institut für Biologie, Berlin-Dahlem.

$I^{\mathrm{N}}$ $\mathrm{N}$ this communication it is proposed to give an exposition of the subject of the determination of sex presenting chiefly the line of argument which the writer has been able to develop from recent work on the question. In doing so it will be convenient to confine our attention to one line of thought, though this will compel us to omit mention of much important work upon the problem. Further, it is proposed to limit the account to the writer's own field of work-namely, the animal kingdom. For a more complete account we refer the reader to the author's book, "Mechanismus und Physiologie der Geschlechtsbestimung" (Borntraeger, Berlin, 1920).

The situation in regard to sex which is typical in nature is that out of a number of fertilised eggs of a given species about equal numbers of male and female individuals are developed. The problem of the determination of sex, then, presents itself in the form of two principal questions : first, what is the mechanism which, at a certain moment, separates the flow of development into two different streams-those of female and male differentiation; and secondly, what is the material difference in the two sets of individuals thus separated, and how does the supposed difference act physiologically in order to direct individual development along female or male lines? We may call the first of these questions the problem of the mechanism of distribution of the two sexes; while the second is the problem of the physiology of sexual determination.

It will be clear to every student of biology that the first problem in question is part of the general problem of the mechanism of heredity-i.e. it is concerned with the transmission of genetic properties from parent to offspring and their distribution among offspring. Therefore the study of the mechanism of distribution of the sexes has formed an integral part of modern work in genetics, and partaken of its triumphal progress. We may safely say that to-day, in the light of Mendelism and the work accomplished in the realms of cytology, the problem is solved as completely as the methods of biology permit.

The first successful attack upon the problem was made when Doncaster and Raynor discovered and studied the famous case of sex-linked inheritance in the currant-moth, and Bateson and Punnett furnished the Mendelian analysis of the case. By following the hereditary distribution of a somatic character closely linked with the distribution of sex, the inference could be drawn that one sex must be heterozygous for a Mendelian factor connected with sexual differentiation, and the other sex homozygous. Thus one sex produces two kinds of gametes in respect to the factor in question, the other sex only one kind. The resulting situation is, therefore, the same as in a back-cross between a hybrid $\mathrm{A} a$ and the pure recessive form $a a$; both types reappear again in No. 2703 , VOL. IO7? the offspring in equal numbers. Since then an immense number of cases of sex-linked inheritance have been analysed, all with the same general result; one sex is homozygous in regard to a sexdifferentiator, and produces one type of gametes -i.e. it is homogametic; the other sex is heterozygous, and produces two types of gametes-i.e. it is heterogametic. There is one complication so far as certain groups of animals are concerned: in mammals and in most of the insects the male is the heterozygous sex, whereas in moths and birds it is the female which produces the two kinds of gametes. The possible meaning of these two types is, however, a question of detail which does not concern us here.

Almost simultaneously with the solution of the problem of the mechanism of distribution of sex in terms of Mendelian symbolism, McClung announced that the odd chromosome found in the sperm-cells of certain Orthoptera and Hemiptera might act as a differentiator of sex. Since then the study of the sex-chromosomes has progressed with a rapidity and success which have rivalled Mendelian discoveries regarding sex. The simple result which stands out to-day as one of the basic facts of cytology is this: all the cells of the body of many animals contain in one sex either an odd chromosome, called an X-chromosome, or an unequal pair of chromosomes, called an $X-Y$ group. The cells of the other sex contain, instead, two X-chromosomes. As is well known, all sexcells undergo a reduction division which reduces the somatic number of chromosomes to one-half; this reduction is brought about by a pairing of each maternal with a corresponding paternal chromosome and subsequent disjunction of whole chromosomes during the meiotic division. An odd $\mathrm{X}$-chromosome, whether it has a $\mathrm{Y}$-partner or not, must, therefore, pass undivided to one of the daughter-cells during the meiotic division. The result is the production of two mature sexcells, one with $\mathrm{X}$, the other without $\mathrm{X}$. In other words, the sex containing the odd $X$ (or the $X-Y$ group) forms two kinds of gametes, which are with and without $\mathrm{X}$ respectively-i.e. it is heterogametic. The other sex, however, with its two X's, produces only gametes with $\mathrm{X}$, and is therefore homogametic. In fertilisation, then, an $\mathrm{X}$-gamete of the latter sex may unite either with a Y-gamete, or with an X-gamete of the heterogametic sex. The result is XX- and XYzygotes-i.e. the two sexes.

The close parallelism between the genetic and cytological facts led Gulick, Morgan, and the writer to venture the opinion that the genetic facts of sex-linked inheritance could be completely explained, if it were assumed that Mendelian factors which are inherited in that peculiar way are carried within the X-chromosomes. Such an assumption would lead to the view that the Mendelian explanation of sex-linked inheritance and 
distribution of sex is only a symbolical way of representing what actually happens when the mechanism of the $\mathrm{X}$-chromosomes is set to work; or, as we put it occasionally, both sets of facts express the same thing in different language.

Recent work has proved the correctness of such assumptions. We need mention only that in the fly Drosophila, where breeding work showed the male to be the heterozygous sex, cytological investigation also demonstrated the existence of an $\mathrm{X}-\mathrm{Y}$ group in the male (Morgan and collaborators); in moths, where genetic proof exists that the female is the heterozygous sex, the existence of an odd $\mathrm{X}$-chromosome in the female was conclusively shown (Seiler). But what we may regard as final proof was furnished by Bridges when he analysed cases in which unexpected genetical behaviour of sex-linked characters was shown to be explicable on the assumption of a non-disjunction of sex-chromosomes during the meiotic division, and when he was able to add cytological evidence of such an event to the genetic proofs. Thus we are led to believe that the mechanism of the distribution of the two sexes among the offspring is perfectly known; it is furnished by the distribution during meiotic division of the sexchromosomes, carrying, among other factors, the sex-differentiators. We are confident that the little opposition which is still encountered occasionally will soon vanish before the weight of facts in favour of such conclusions.

A knowledge of the mechanism at work is a safe basis from which we may attack the second part of the problem of sex and so find an answer to the question: How does the one-X-two-X mechanism act physiologically in order to secure the differentiation of one or the other sex? The first attack upon this problem has been made by analysing a phenomenon which we have termed "intersexuality," and the main line of the facts and the analysis in question are given below.

The work was done with the gipsy-moth, in which the female is the heterogametic sex and the mechanism of the distribution of sex is perfectly normal. The phenomenon of intersexuality occurs, then, as breeding experiments show, without any disturbance of this mechanism. Intersexes-i.e. individuals which show definite mixtures of the characters of both sexes, and, as a whole, appear to occupy a definite position between the two sexes-are produced regularly and at will in crosses between different geographic races of the gipsy-moth. If, for example, a female of the Japanese race from Tokyo is crossed with a South European male, all the offspring are normal; in the reciprocal cross, however, all males are normal, but all would-be females intersexual. Or, again, if we cross a female of a Japanese race from Hokkaido with a male from Fukuoka, all the offspring are normal, but in the reciprocal cross all females are normal and all would-be males intersexual.

If we fix our attention, for the sake of simplicity, only on the intersexual females-i.e. interNo. 2703 , VOL. IO7] sexes with the factorial and chromosomal constitution of a female-we may state that the majority of the different races belong to one of two categories-first, what may conveniently be termed weak races; and secondly, strong races, which are those the males of which, if crossed with the female of a weak race, produce normal males and intersexual females. In testing the different strong races at our disposal in crosses with females of any particular weak race, we find among the strong races a graded series according to "strength." The males of one strong race produce with the weak female a low type of intersexuality, individuals which exhibit only slight addition of maleness to their female constitution. Another strong race produces with the same race of females a higher type of intersexuality; still another may produce.a high grade of intersexual females; while a fourth may finally transform all would-be females into males, which cannot be distinguished (except by breeding tests) from genetic males. If we test the different weak races by crossing their females with any particular race of strong males, we find again a series of degrees of weakness as shown by the lower or higher type of resulting intersexuality. From such experiments it follows that female intersexuality is produced if a female of a weak race is crossed with a male of a strong race; further, that the grade of intersexuality depends upon two variables-viz. the relative degrees of weakness and strength of the parental races; in other words, it depends upon a quantitative relation of what we have termed weakness and strength.

By applying breeding tests it was shown further that strength follows in inheritance the distribution of the X-chromosomes or the sex-factor. Strength must therefore be regarded as a property of the well-known Mendelian sex-factor located in the X-chromosome. What we have termed weakness, however, is inherited purely maternally. This may mean that it is transmitted within the protoplasm or the $\mathrm{Y}$-chromosome, and in any event it must be equally present in every egg. All these facts show clearly that an explanation on ordinary Mendelian lines is not possible. Something has to be added to ordinary Mendelian symbolism in order to account for the facts, and this addition is the assumption that the factors in question are possessed of a definite valency which acts in a quantitative way.

The X-chromosome contains the factor for maleness, whereas the factor for femaleness is inherited maternally. The quantity of the latter is constant for each egg, whereas the quantity of the former is double in the male $(\mathrm{XX})$, single in the female (X). If there exists such a normal relation that the one male quantity is less efficient than the female quantity, while two male quantities act more strongly than the constant female quantity, and, further, if it be assumed that the higher quantity controls sexual differentiation, it is obvious why normally one or the other sex is produced, although each egg might, 
as experiments show, develop into a female, a male, or something between. Finally, if it be assumed that the strong races are possessed of sex-factors of a higher absolute quantity, the production of intersexuality in the crosses is also explained-the big dose of a male factor confronted as the result of crossing with a dose of the female factor which is relatively too small determines the character of the offspring even in the one-dose (X) state. As a matter of fact, all the breeding experiments devised to test such views have given results in accordance with theory.

Fortunately, the analysis of the intersexual individuals could be pushed one step further towards a physiological understanding. It could be demonstrated by a very large number of really amazing morphological and embryological facts that intersexual females are individuals which had developed up to a certain moment as females, when suddenly the sex had changed and development was finished as a ma $1 \mathrm{e}$. Similarly, intersexual males begin as males and end as females, and the different types of intersexuality were proved to be the consequence of the position of the turning point in development. A late turning point means that only certain organs, which have not completed their development, can be forced into the line of differentiation of the other sex; the result is an intersex of low grade. An earlier position of the turning point consequently leads to the production of the higher grades of intersexes, and a still earlier position to the complete reversal of sex. The degree of intersexuality is inversely proportional to the position of the turning point in the progress of development. The position at this point of the analysis is this: on one hand we have the presence of characteristic doses of substances called sex-factors in definite quantities; on the other, there is a period of varying duration (the time of development up to the turning point), the length of which is proportional to the difference in the quantities of the two sex-factors. This points emphatically to the idea that the sex-factors are substances which cause, take part in, or accelerate a reaction in proportion to the quantity present. The result may then be represented in the graph (Fig. I): on the abscissa is plotted the time of development, the line $t-t$ being the end of embryonal and larval differentiation. The ordinate indicates the amount of that product of the activity of the sex-factors which carries differentiation in the direction of one sex. The curve $F$ shows the rate of production of the femaleNO. 2703 , vOL. $\left.\mathrm{IO}_{7}\right]$ determining substances, which is constant for each egg of a given race. $\mathrm{Mm}$ is the curve for the male-determining substances in the female (one dose, X); $M M$ in the male (two doses, XX). In normal reproduction the $\mathrm{F}$ and $\mathrm{M}$ curves do not intersect in development. $\mathbf{M}_{1} m, \mathbf{M}_{2} m$, etc., are the curyes of the male-determining substances produced by the larger quantities of $M$ substance in the $\mathrm{X}$-chromosomes of the strong races. Their points of intersection with the $F$ curve (in the case of hybrid combination) occur during development, and represent the turning point where sex changes from femaleness to maleness. The graph then gives the physiological solution of the case of intersexuality; simultaneously it answers the question which led to the consideration of the work on intersexuality-viz. : How does

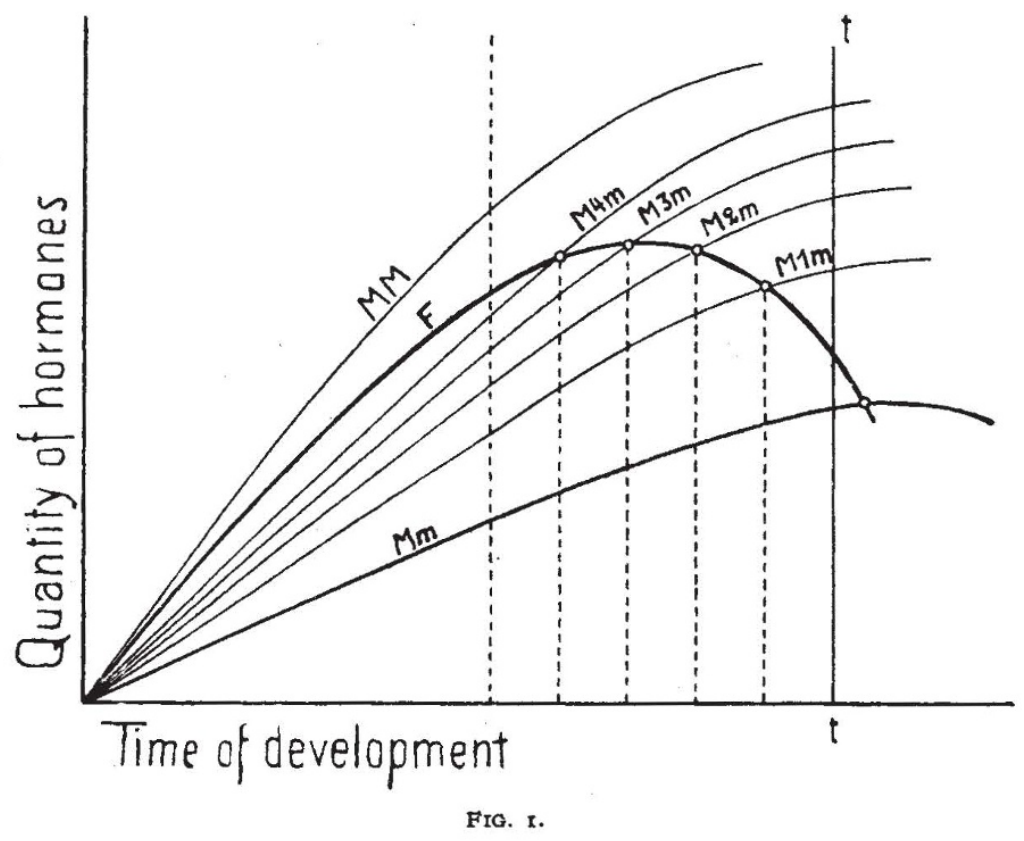

the presence of one or two X-chromosomes containing sex-factors act physiologically in order to induce the differentiation of one or the other sex? The answer is: The mechanism which produces germs with two and one $\mathrm{X}$ respectively is an ideal mechanism to secure the higher velocity to one or the other of two simultaneous and competing reactions, namely, the male and female reaction, by starting it with the greater quantity of reacting substance.

But there is a limit to our analysis so far as the work on intersexuality is concerned. We can see no means of ascertaining in moths in what this reaction, the velocity of which is influenced by the concentration of the reacting substances, really consists. The answer can be given, we believe, by the facts of hormonic intersexuality.

It is well known to every student of biology and physiology that in the higher vertebrates, at least in birds and mammals, the endocrine function of the sex-gland plays an important rôle in the development of secondary sex-characters. 
Early castration in mammals prevents the normal development of the visible characters, and results in the assumption even of female secondary characters by male birds. Early and successful transplantation of the heterologous gonad makes either sex assume, to a more or less complete degree, the characters of the other sex (Steinach, Goodale, etc.). We might term this the production of hormonic intersexuality, but, of course, changes appear only in those organs which are able physiologically to change under the influence of hormones, irrespective of the genetic constitution in regard to sex. But the methods which have to be used exclude a complete experiment in intersexuality, where the entire body, including sexglands, ducts, etc., must react. Fortunately, Nature has performed such an experiment for us, as the recent analysis of the case of the "freemartin," which we owe to the work of Keller, Tandler, and Lillie, has revealed. Among twin calves, cases of normal male and female are very rare. If both are not of the same sex, in most cases a normal male is accompanied by an abnormal hermaphrodite female, the freemartin. It is now known that this freemartin is a typical case of hormonic intersexuality. The authors quoted above have been able to show independently that in this case-but in this case alonean anastomosis between the blood-vessels of the twins occurs, so that the same blood flows through both. In the male partner the testis, with its interstitial tissue, develops first, and before the ovary of the female has reached the stage of endocrine function. So the female comes under the influence of the male hormones, the ovary stops differentiation, and all the sex-characters develop in the male direction. The result is the freemartin, a calf with female external sexorgans, almost male sexual ducts, and a sex-gland containing sperm tubules which are incapable of spermatogenesis. Most interesting corroboration of this interpretation has recently been furnished in Lillie's laboratory by Minoura, who was able to produce hormonic intersexuality experimentally by transplanting gonads into developing chickens' eggs.

If we compare this case of true hormonic intersexuality with the zygotic intersexuality of the moths, we see at once that the "turning point" from, which sexual differentiation changes in the intersexual moth corresponds exactly to the moment when the male hormones are poured into the blood of the female in the case of the freemartin. Comparing the facts carefully, we feel justified, therefore, in giving the following answer to our former question: What is this reaction which is accelerated by the action of the sex-substances with a velocity proportional to their concentration? The reaction is the production of the specific hormones of sexual differentiation. In insects this occurs in every cell of the body as an irreversible consequence of the combination in fertilisation. In the higher vertebrates the reaction becomes more or No. 2703 . VOL. IO7] less centralised within the interstitial tissue of the sex-glands.

That this solution of the problem of sex comes near the truth is rendered probable by the ease with which even the most complicated sexual phenomena fall in line with the theory. The questions of parthenogenesis and sex, sex-mosaics or gynandromorphs, sexual polymorphism, inheritance of secondary sex-characters, and the different types of hermaphroditism, all find simple solutions, or, at least, appear capable of such. This may be demonstrated in the interesting case of the Gephyrean worm Bonellia, well known for its extreme sexual dimorphism, the male being a rudimentary microscopic worm which lives as a parasite in the oviduct of the large female. Baltzer made the discovery that part of the larvæ, developed from fertilised eggs, become attached to the proboscis of an adult female, and live there for some time in a semi-parasitic way before developing into males. Larvæ, however, which undergo development without the parasitic stage remain for some time undifferentiated, and then develop into females. If larvæ which are fixed to the proboscis of a female are removed after a shorter or longer period, intersexes of different type are produced. Let us now suppose that we could devise an experiment to prove directly the correctness of the quantitative view of sex-determination as represented in the above graph. We might perform it successfully by finding a method of accelerating or retarding the rate of differentiation without influencing the rate of the production of the sex-hormones. In the event of success we ought to be able to shift the point of intersection of the $F$ and $M$ curves back into the time when differentiation was still in progress. The result would be intersexuality. It seems that Bonellia is able to perform this experiment by means of the excretion of her proboscis. The $\mathrm{F}$ and $\mathrm{M}$ curves of the larvæ seem to have such a relation that the male hormones are being produced quickly, and the female hormones slowly. The normal rate of differentiation is slow-so slow that sexual differentiation begins only when the phase of action of the male hormones has passed, and females are produced exclusiveiy. The secretion of the proboscis, however, accelerates the rate of differentiation in a way analogous to the action of the thyroid in accelerating metamorphosis in amphibians. In the case of parasitism of the larvæ, therefore, differentiation takes place during the phase of action of the male hormones. Interruption of the influence of the secretion naturally causes intersexuality. Finally, we may state that recently we were successful to a certain extent in imitating this experiment with moths. By employing low temperatures we could put back the turning point for females of pure races of the gipsy-moth and thus produce intersexuality.

Ever since genetics assumed its modern form the problem of sex has been closely linked with the general problem of heredity. The Mendelian study of sex formed part of the general study of 
genetic factors, while the cytological study of sex was closely connected with the chromosome-theory of Mendelian heredity. It therefore appears rather tempting to apply the quantitative views of sexual differentiation to the theory of heredity in general. Recently an attempt has been made by the writer in "Die quantitativen Grundlagen von Vererbung und Artbildung" (Berlin, J. Springer, I920) to attack the problem of the physiology of heredity from this point of view. A discussion of this would, however, be beyond the scope of this contribution.

\section{Further Remarks on Relativity. ${ }^{1}$}

\section{By Sir Oliver Lodge, F.R.S.}

III.

\section{Changes of Frequency.}

$\mathrm{A}^{\mathrm{c}}$ CCORDING to the usual presentation of relativity, clocks appear to go slow to a relatively moving observer; quite irrespective of any Doppler effect, which can readily be allowed for. Their rate would have to be multiplied by the fraction $I / \beta$ or $\sqrt{ }\left(\mathrm{I}-u^{2} / c^{2}\right)$; which means that a clock on the sun seen from the earth, say on December $3 \mathrm{I}$ or July I when the motion is exactly transverse, would lose one second in two hundred million, or about sixteen seconds per century.

But, for testing purposes, we cannot change the motion appreciably, and so we cannot hope to tell if the clock would seem to go quicker if we stopped. Reversal of motion, even if it could be accomplished, would be no good; the difference to be observed-unlike the Doppler effect-is between motion and rest, or between rapid motion and slow, not between plus and minus motion. If we had a clock which we could fix at relative rest to ourselves, and yet be sure that it kept time with the one we were observing on some relatively moving body, the comparison might be made. And the revolution or vibration of a radiating atom, $(a)$ on earth, and $(b)$ on sun or star, appears to satisfy the conditions. If source and observer were moving together, there would be compensation; but if either was moving without the other, there should be an effect, such as by long accumulation might be detected. The Mercury effect allowed accumulation for a century or more. The spectrum effect does not allow any accumulation; whatever can be seen there must be seen instantly, it must depend on what happens in a single period. It is true that a certain train of waves is needed for visibility, and some succession is necessary for interference; but so short is the series required for interference that position in the spectrum is practically dependent on individual wave-length.

The value of $u^{2}$ for the earth's orbit, considered circular, is equal to the sun.'s gravitational potential at the earth's distance under the inverse square law, say $-\mathrm{V}$; or twice that potential under the direct distance or centrifugal-force law. Hence the slowness to be expected, $\sqrt{ }\left(\mathrm{r}-u^{2} / c^{2}\right)$, may be written either $\mathrm{I}+\mathrm{V} / 2 c^{2}$ or $\mathrm{I}+\mathrm{V} / c^{2}$; and the second term is the displacement towards the red which is being looked for. Only, of course, it is being looked for where the potential is strongest, viz. close to the sun; for there it is two hundred times stronger than in the neighbourhood of the 1 Continued from p. 751 . earth (the radius of the earth's orbit being two hundred times the radius of the sun). It seems, however, that a small fraction of the gravitational effect ought to be produced as the result of the earth's motion, even if the sun were nothing but a central source of light.

The occurrence of the factor 2 is curious, and corresponds with a similar factor in the raybending calculation. But I do not now discuss it, because a spectral shift due to transverse motion is doubtful. Space-measuring rods shrink, it is true, but in the direction of motion, not in the sun's direction; so the measured velocity of light from the sun would be constant without any timecorrection. Yet it is not easy to see how a clockdiscrepancy can be dependent on the direction of motion, apart from the ordinary allowance for light-speed.

\section{Changes of Inertia and Weight.}

That an electric charge possesses the fundamental material quality of inertia, by reason of the magnetic field which inevitably is generated when it moves, was first calculated by Sir J. J. Thomson so long ago as $188 \mathrm{I}$. That this electrical inertia is a function of speed, so that as the speed of light is approached it ought to undergo a rapid increase of value, was predicted, and its amount reckoned, by both J. J. Thomson and Oliver Heaviside. That the facts of observation were in accord with the prediction, was verified, first by Kaufmann and then by others; while that this subordinate dependence of inertia on speed applies even to neutral atoms of matter, is a consequence of the fairly ascertained electrical nature of their constitution. On the theory of relativity the variation of inertia appears to follow, without any electrical theory at all, as a result of changing the frame of reference to moving axes. The additional mass corresponds to the kinetic energy of the moving matter divided by $c^{2}$. Which suggests that the whole mass is probably a demonstration and a result of fine-grained ætherial rotational energy with velocity $c$.

It is legitimate, anyhow, to assume as a working hypothesis that the mass of a body is not really constant, but that at the speed $u$ it becomes $m=\beta m_{0}$ or $m_{0} / \sqrt{ }\left(\mathrm{I}-u^{2} / c^{2}\right)$.

The speed necessary to display this effect is usually attained only by electrons and positive nuclei in a vacuum tube, or by aid of spontaneous radio-activity; but the refinements of astronomy are so great that the planet Mercury is moving fast enough to exhibit some result dependent on NO. 2703 , VOL. IO7] 